\title{
Evaluación de mutaciones en los genes GJB2 y GJB6 en pacientes con sordera congénita identificados mediante screening neonatal
}

\author{
GABRIELA LÓPEZ V. ${ }^{1}$, ALONSO PUGA Y. ${ }^{2}$, ENRICA PITTALUGA P. ${ }^{3}$, \\ LORETHO BUSTAMANTE M. ${ }^{4}$, CRISTIÁN GODOY B. ${ }^{4}$, M. GABRIELA REPETTO L. ${ }^{5}$ \\ 1. Estudiante de Medicina, Facultad de Medicina, Clínica Alemana-Universidad Del Desarrollo. Santiago de Chile. \\ 2. Bioquímico, Centro de Genética Humana, Facultad de Medicina, Clínica Alemana-Universidad Del Desarrollo. \\ Santiago, Chile. Actualmente en Centro de Genómica y Bioinformática de la Universidad Mayor. \\ 3. Médico Neonatólogo, Complejo Hospitalario Dr. Sótero del Río. Santiago de Chile. \\ 4. Fonoaudiólogo, Complejo Hospitalario Dr. Sótero del Río. Santiago de Chile. \\ 5 Médico Genetista, Facultad de Medicina y Centro de Genética Humana Clínica Alemana-Universidad Del Desarrollo. \\ Santiago, Chile.
}

\begin{abstract}
Evaluation of GJB2 and GJB6 gene mutation in Chilean patients with congenital deafness identified through neonatal screening

If not detected and treated early, congenital sensorineural hearing loss generates impairment in linguistic, intellectual and social development of individuals. Most congenital hearing deficits are genetic. The most common causes are mutations in GJB2 and GJB6 genes, both located on chromosome 13, encoding junction proteins that allow the transduction of sound in the inner ear. Objetive: To evaluate the presence of mutations in GJB2 and GJB6 genes in a population of children diagnosed with deafness in Complejo Hospitalario Sótero del Río since implementation of the universal newborn hearing screening program. Patients and Methods: 8 patients with congenital nonsyndromic sensorineural deafness were evaluated. Genomic DNA was extracted from oral mucosa swabs. PCR was performed to identify the 35delG mutation in GJB2, followed by sequencing of this gene, and PCR for 2 GJB6 deletions. Results: Two patients were heterozygous for 35delG mutation in GJB2, being their other alleles normal. Another 2 patients were heterozygous for V27I polymorphism, one of them also accompanied by p.A148A (c.444C > A) variant. A patient was found with a previously undescribed mutation (c.4360 C>T) in GJB2's intron 1, being the second allele normal. No mutations were identified in GJB6. Conclusions: In this population of children, mutations in the GJB2 gene were an identifiable cause of congenital sensorineural
\end{abstract}

(Key words: GJB2, GJB6, Congenital sensorineural hearing loss).

Rev Chil Pediatr 2012; 83 (2): 154-160

Trabajo recibido el 17 de agosto de 2011, devuelto para corregir el 02 de enero de 2012, segunda versión 14 de enero de 2012 , aceptado para publicación el 03 de febrero de 2012.

Financiamiento: Concurso de Investigación del Departamento Científico-Docente de Clínica Alemana (G.R.) y FONIS, proyecto SA04i2032 (E.P.)

Correspondencia a:

Dra. M. Gabriela Repetto L.

E-mail: grepetto@udd.cl 


\section{RESUMEN}

La hipoacusia neurosensorial congénita es una patología frecuente que si no es detectada y tratada oportunamente genera alteraciones en el desarrollo del niño. Desde el año 2005 se lleva a cabo en el Complejo Hospitalario Dr. Sótero del Río un programa de screening auditivo universal para la detección precoz de esta patología. La mayor parte de los déficits auditivos congénitos son genéticos. La etiología más común son las mutaciones en los genes GJB2 y GJB6, que codifican para proteínas “gap junction” que permiten la traducción del sonido en el oído interno. Objetivo: Evaluar la presencia de mutaciones de los genes GJB2 y GJB6 en una población de niños diagnosticados con hipoacusia congénita en el Complejo Hospitalario Dr. Sótero del Río a través del programa de screening auditivo universal. Pacientes y Método: Se evaluaron 8 pacientes con hipoacusia congénita neurosensorial no sindrómica. Se extrajo ADN genómico de hisopado de mucosa bucal y se realizó PCR para identificar la mutación 35delG en GJB2, seguida de secuenciación de este gen, y PCR para 2 deleciones del gen GJB6. Resultados: Dos pacientes fueron heterocigotos para la mutación 35delG en GJB2, siendo sus otros alelos normales. Dos fueron heterocigotos para el polimorfismo V27I; uno acompañado por la variante p.A148A (c.444 C > A). Se encontró además un paciente con una mutación no descrita anteriormente (c.4360 C>T) en el intrón 1 de GJB2, siendo su segundo alelo normal. No se identificaron mutaciones en GJB6. Conclusiones: En este grupo de niños estudiados se encontró mutaciones en el gen GJB2, causantes de sordera neurosensorial congénita.

(Palabras clave: GJB2, GJB6, hipoacusia neurosensorial congénita).

Rev Chil Pediatr 2012; 83 (2): 154-160

\section{Introducción}

Se define hipoacusia como una pérdida auditiva con un umbral mayor de $20 \mathrm{~dB}$ uni o bilateral, la cual puede ser neurosensorial, conductiva o mixta. La incidencia de hipoacusia neurosensorial (HNS) congénita es 1 a 3 de cada 1000 recién nacidos vivos ${ }^{1-3}$.

Dado el impacto de la pesquisa y tratamiento precoz de la HNS, idealmente en la etapa prelingual, varios países han iniciado programas de tamizaje o "screening" auditivo neonatal. Los test de screening incluyen la respuesta auditiva automatizada de tronco cerebral (AABR) y las emisiones otoacústicas (EOA) ${ }^{4}$. Este screening nace de la necesidad de implementar tempranamente medidas para mejorar las habilidades lingüísticas y calidad de vida de los pacientes que sufren esta enfermedad, considerando que la implementación de estímulos auditivos durante los primeros 6 meses de vida es fundamental y crítica en el desarrollo de habilidades del lenguaje ${ }^{5}$. El diagnóstico y tratamiento de la HNS habitualmente es retrasado hasta la edad de dos años a tres años cuando no existen programas de tamizajes o screening auditivo neonatal.

En el año 2005, en Chile se implementó el programa de detección precoz de sordera en la población de prematuros menores de 32 semanas o menores de 1500 gramos.

Concomitantemente, en abril de 2005 se implementó en el Complejo Hospitalario Dr. Sótero del Río el programa de screening auditivo universal ${ }^{6}$. Diversas organizaciones han recomendado la inclusión del estudio etiológico de los recién nacidos con sordera, dada la relevancia del consejo genético e identificación de personas en riesgo en los casos de hipoacusia de causa hereditaria.

La mitad de los casos de sordera se atribuyen a factores genéticos y la mitad ambientales. Las causas adquiridas comúnmente resultan de infecciones prenatales como la provocada por los organismos del síndrome de TORCH (toxoplasmosis, rubeola, citomegalovirus, y virus herpes). Más aun, en países desarrollados la causa más común es representada por la infección congénita de citomegalovirus, con un prevalencia total al nacimiento de $0,64 \%$. Entre las causas genéticas, un tercio se deben a síndromes (Down, Treacher Collins, etc), de los cuales hay más de 400 tipos ya descritos. Un $70 \%$ se clasifican como no sindrómicos, por lo que no hay signos físicos externos que alerten a la existencia de hipoacusia. La forma de transmisión autosómica recesiva es la más frecuente entre las causas no sindrómicas (75- 
$85 \%$ ) y entre estas, se estima que un $50 \%$ se debe a mutaciones en genes que codifican para conexinas en el oído interno.

Se ha demostrado que las uniones intercelulares gap junction a nivel de las células ciliadas internas juegan un rol fundamental en la homeostasis de iones y electrolitos en el oído interno, especialmente en el rol de reciclar potasio en la cóclea, permitiendo así el mecanismo de transducción del sonido. Los gap junction están formados por un gran número de conexones, que son pequeños canales proteicos formados por 6 proteínas transmembrana, llamadas conexinas ${ }^{7}$. Las mutaciones en los genes codificadores de las conexinas pueden alterar la función de las proteínas del oído interno resultando en sordera no sindrómica congénita ${ }^{8}$.

La mutación más común es la deleción de la guanina en la posición 35 en el gen GJB2 (35delG), localizado en el locus DFNB1 del cromosoma 13, el cual codifica la conexina $26^{9-14}$. Esta mutación ha sido reportada como causa de un 50\% de la sordera pre lingual recesiva en caucásicos. En estas poblaciones, un $10-50 \%$ de los pacientes presentan esta mutación en forma homocigota recesiva y otro 50\% heterocigota. Estos últimos pacientes pueden tener otra mutación en el otro alelo de GJB2, o una deleción (del) del gen adyacente GJB6 el cual codifica para la conexina 30: del(GJB6D13S1830) o del(GJB6-D13S1854). Estas deleciones son comunes en pacientes españoles con hipoacusia ${ }^{8,15,16}$.

Pese al interés de la pesquisa y rehabilitación precoz de la hipoacusia neurosensorial congénita, las causas de sordera congénita en nuestra población son desconocidas. El objetivo de este estudio fue evaluar la presencia y frecuencia de mutaciones en GJB2 y deleciones en GJB6 en un grupo de niños diagnosticados con hipoacusia neurosensorial moderada y severa en el Complejo Hospitalario Dr. Sótero del Río.

\section{Pacientes y Método}

Evaluamos a pacientes con hipoacusia congénita neurosensorial (HNS) no sindrómica moderada a severa derivados del programa de screening auditivo neonatal y diagnosticados en el servicio de otorrinolaringología en el Complejo Hospitalario Dr. Sótero del Río en el período 2005-2008 ${ }^{6}$.

Previo consentimiento informado, se extrajo ADN genómico de hisopado de mucosa bucal fijado en tarjeta FTA Card $®$ (Whatman, Florham Park, NJ. EE.UU.) según protocolo del fabricante. Se realizó la reacción en cadena de la polimerasa (PCR) alelo-específica utilizando los partidores y las condiciones descritas por Scott et al $^{11}$, para identificar la mutación 35delG en GJB2, seguida de secuenciación bidireccional de los exones 1 y 2 y los bordes intrón-exón de este gen, utilizando secuenciador automático ABI3730. Finalmente, se realizó PCR para las 2 deleciones del gen GJB6: del(GJB6-D13S1830) y del(GJB6-D13S1854), utilizando los partidores y condiciones descritas por del Castillo FJ et $\mathrm{al}^{8}$ (figura 1 ).

\section{Resultados}

Diecisiete niños fueron identificados con HNS bilateral congénita moderada a severa, de una población de 23856 recién nacidos sometidos a screening auditivo neonatal entre 2005 al 2008 en la Maternidad y Neonatología del Hospital Sótero del Río, es decir, una incidencia de 0,72/1 000. De estos, 8 participaron en el estudio. Los 9 restantes presentaban hipoacusia en relación a síndromes específicos o anomalías asociadas como fisura palatina, por lo que fueron excluidos de este estudio. De los 8 niños incluidos, tres tenían HNS moderada y 5 , profunda. Para esta división se utilizó la clasificación americana de sordera, la cual muestra que un grado leve equivale a una pérdida entre 20-40 dB, moderado entre 41-60 dB, 61-80 dB severa y una pérdida de la audición sobre $80 \mathrm{~dB}$ se denomina profunda ${ }^{21}$.

Los genotipos de los pacientes y su diagnóstico clínico se encuentran resumidos en la tabla 1. Encontramos 2 mutaciones conocidas, una potencial mutación nueva y dos polimorfismos o variantes normales. Durante el estudio inicial realizado con PCR-AS (figura 2), se demostró que dos pacientes tenían un alelo con 


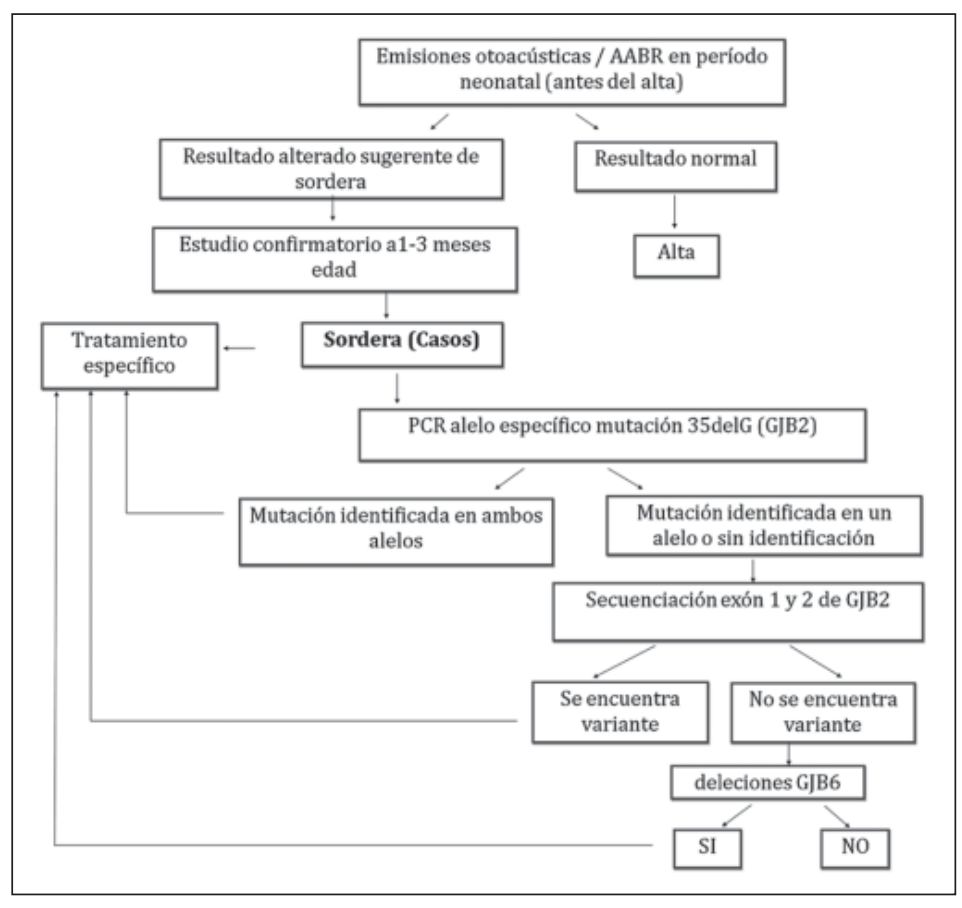

Figura 1. Metodología de estudio genético para GJB2 y GJB6 de pacientes con HNS. HNS: hipoacusia neurosensorial. AABR: Automated auditory brainstem response (Respuesta auditiva automatizada de tronco cerebral). PCR: Polymerase chain reaction. Mapa conceptual que muestra la metodología de estudio genético para GJB2 y GJB6, pacientes que dieron positivo en el screening neonatal de hipoacusia procedieron a un estudio confirmatorio a los 1-3 meses, finalmente se obtuvieron 8 casos de sordera a las que se le aplicó estudio genético mediante PCR para la mutación GJB2, si el pacientes no presentaba dicha mutación o era heterocigoto para ella, se procedió a secuenciar el exón 1 y 2 del mismo gen. Posterior a ello se procedió finalmente a la búsqueda de las 2 deleciones del gen GJB6: del(GJB6-D13S1830) y del(GJB6-D13S1854).

Tabla 1. PCR dels

\begin{tabular}{|c|c|c|c|c|c|c|}
\hline Casos & & Fenotipo & $\begin{array}{c}\text { del(GJB6- } \\
\text { D13S1830) }\end{array}$ & $\begin{array}{c}\text { del(GJB6- } \\
\text { D13S1854) }\end{array}$ & $\begin{array}{l}\text { PCR-AS- } \\
\text { 35delG }\end{array}$ & Secuenciación \\
\hline 1 & CX-049 & HNS b. Profunda & $-/-$ & $-/-$ & $-/-$ & V27I y A148A (c.444 C > A) \\
\hline 2 & CX-050 & HNS b. Profunda & $-/-$ & $-/-$ & $-/-$ & SV \\
\hline 3 & CX-051 & HNS b. Profunda & $-/-$ & $-/-$ & $-/-$ & SV \\
\hline 4 & CX-052 & HNS b. Profunda & $-/-$ & $-/-$ & $+/-$ & 35delG \\
\hline 5 & CX-053 & HNS b. Profunda & $-/-$ & $-/-$ & $+/-$ & 35delG \\
\hline 6 & CX-054 & HNS b. Moderada & $-/-$ & $-/-$ & $-/-$ & V27I / - \\
\hline 7 & CX-055 & HNS b. Moderada & $-/-$ & $-/-$ & $-/-$ & g.4360 C > T (intrón 1) \\
\hline 8 & CX-056 & HNS b. Moderada & $-/-$ & $-/-$ & $-/-$ & SV \\
\hline
\end{tabular}

HNS b.: Hipoacusia Neurosensorial bilateral. SV: Sin variante. "-: sin mutación o deleción. +: con mutación o deleción.

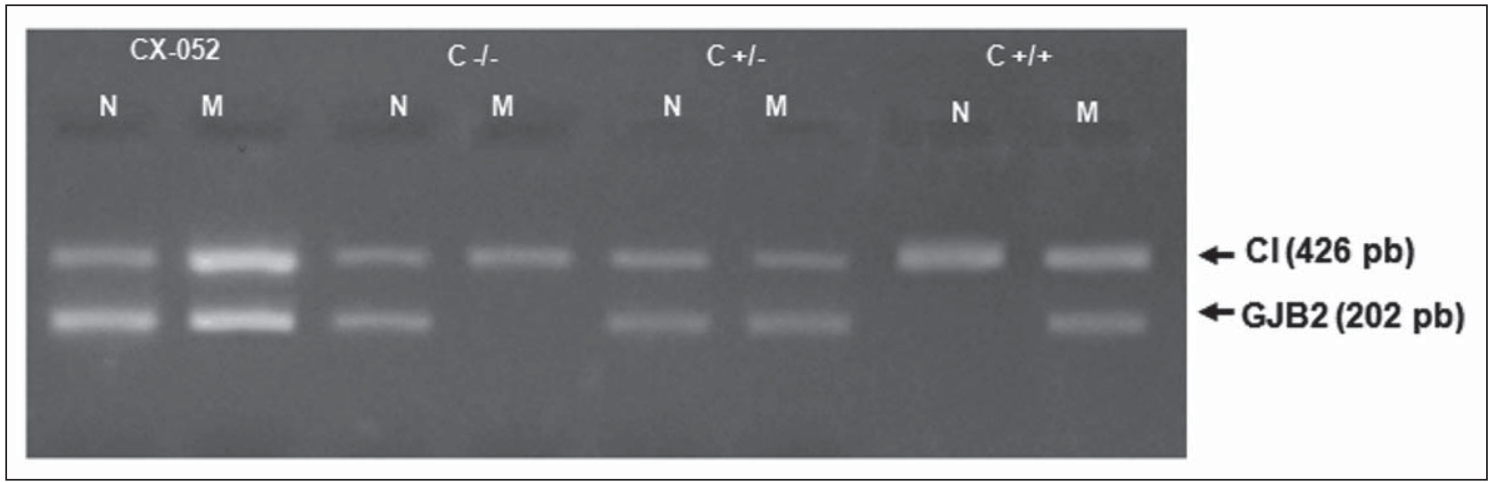

Figura 2. Electroforesis. CI: control interno. N: normal. M: Mutado. C-/-: paciente sin mutación en alelos, al no dibujarse banda en la columna de alelo mutado (M). C+/-: paciente con un alelo mutado, al presentar bandas en las 2 columnas $\mathrm{N}$ y M, por lo tanto heterocigoto para la mutación 35delG n GJB2. C+/+: Paciente con ambos alelos mutados. Esta figura muestra la electroforesis en gel de agarosa, del caso CX-052, el cual muestra ser heterocigoto para la mutación 35delG en GJB2. 
la mutación 35delG. Ambos pacientes presentan hipoacusia neurosensorial (HNS) bilateral y profunda. Esta mutación fue confirmada mediante la secuenciación del gen GJB2. La secuenciación del gen GJB2 además permitió identificar la presencia del polimorfismo V27I (sustitución de valina por isoleucina en el aminoácido número 27 de la proteína). Esta es una variante normal y común en la población, no causante de hipoacusia, que se encontró en dos pacientes no relacionados. En el primero de ellos, que presentaba HNS bilateral profunda, se encontró también la mutación p.A148A (c.444 C>A), que no ha sido previamente descrita por lo que se desconoce su rol patogénico. El segundo paciente que tenía HNS bilateral moderada no presentó otra mutación en su otro alelo. Finalmente, un paciente con HSN moderada fue heterocigoto para una mutación nunca antes descrita (c.4360 C> T) en el intrón 1 de GJB2.

En el último paso del estudio se analizaron las deleciones del gen GJB6 mediante PCR, no observándose en ningún paciente estudiado dichas deleciones.

\section{Discusión}

En este estudio observamos una incidencia de hipoacusia congénita levemente menor que la publicada en otros estudios pero de magnitud suficiente como para justificar la existencia de programas de pesquisa, confirmación y tratamiento precoz. Esta menor cifra puede deberse a que un 35 a 40\% de los pacientes con screening auditivo no acudieron a un estudio diagnóstico confirmatorio, por lo que la cifra real es probablemente mayor.

Buscamos mutaciones en los genes GJB2 y GJB6 en 8 pacientes diagnosticados con HSN aparentemente no sindrómica mediante screening neonatal en el Complejo Hospitalario Dr. Sótero del Río. Los resultados demostraron la presencia de mutaciones en 2 y polimorfismos en otros 2 de los 8 pacientes. Además, 2 pacientes tuvieron variantes de significado incierto. Dos de los 16 alelos (12,5\%) estudiados presentaron la mutación 35delG en GJB2. Esto evidencia que las mutaciones en GJB2 contri- buyen a causar hipoacusia congénita en nuestro país.

Estos resultados contrastan con los mostrados en otros estudios como los realizados en Argentina, donde la mutación 35delG se presentó en un 59\% de los alelos patológicos estudiados, o los resultados mostrados en la población española o italiana que dio cuenta de una prevalencia de 55\% y $76 \%$ respectivamente. Esto puede deberse al tamaño muestral pequeño de nuestro estudio, así como a la diferente composición étnica de pacientes estudiadas en estas publicaciones. En apoyo a esta segunda hipótesis, nuestros datos muestran cierta consistencia con los datos obtenidos en otros estudios latinoamericanos, donde los porcentajes son menores a los vistos en población caucásica. Por ejemplo, un estudio de 40 pacientes venezolanos donde se ve una frecuencia de 8 pacientes (20\%) que presentaron la mutación de GJB2 en forma heterocigota, sin mutaciones en GJB6 ${ }^{17}$.

Es importante destacar que en diversos estudios se ha demostrado que los pacientes diagnosticados con mutaciones en conexinas presentan una sordera no progresiva, como lo demostró el estudio transversal de 1531 individuos en 16 países, con sordera autosómica recesiva moderada a profunda, a los que se les realizó un análisis genotípico de GJB2 junto con un estudio audiométrico en el cual se demostró mediante análisis regresivos de umbrales auditivos, una progresión no significativa de su sordera ${ }^{18}$.

La variante V27I es un polimorfismo o variante normal ${ }^{19}$, por lo que no explica la hipoacusia de los pacientes. Los otros dos alelos encontrados A148A (c.444 C>A) (1 alelo) y c.4360 C>T (1 alelo) en GJB2, no han sido previamente descritos y podrían jugar un rol en la etiología de la sordera en pacientes chilenos. Sin embargo, hará falta pruebas funcionales de cada una de estas mutaciones para comprobar si cumplirían o no roles en los hallazgos de sordera. Pese a que la variante 444 es sinónima (no cambia el aminoácido codificado), puede tener algún efecto regulatorio sobre la expresión génica. Un hallazgo interesante es la comparación entre las 2 personas que presentan el polimorfismo V27I, ya que el que lo 
presenta con la variante p.A148A (c.444C>A), presenta HSN profunda en cambio el paciente que lo presenta solo, tiene HSN moderada. A pesar que la hipótesis de herencia digénica de sordera de DFNB1 ha recibido soporte de variadas observaciones, al ser ambas conexinas 26 y 30 parte de las mismas estructuras internas del oído, en este estudio no se identificaron pacientes que presentaran las mutaciones en el gen GJB6 (del(GJB6-D13S1854) y del(GJB6-D13S18530)) en ningún alelo. Por el contrario estas mutaciones tienen una alta frecuencia entre las personas con HNS en España ${ }^{16}$.

Adicionalmente, hay estudios recientes muestran pacientes con mutaciones en el gen GJB2, que dieron resultado normal negativo durante el screening neonatal de emisiones otoacústicas, pero que finalmente expresaron sordera de manera más tardía ${ }^{20}$. De hecho, en el Centro de Genética de la Facultad de Medicina Clínica Alemana-Universidad del Desarrollo hemos encontrado la mutación en GJB2 en varios pacientes de otros centros, con hipoacusia neurosensorial prelingual, pero con screening neonatal normal (datos no publicados), lo cual sugeriría la necesidad de un segundo screening en la infancia.

Por último, sería importante aumentar el número de recién nacidos que se les realiza un screening auditivo, así como el estudio etiológico de aquellos diagnosticados con HSN. El estudio genético de estos pacientes ayudará a comprender la etiología, a estimar las probabilidades de recurrencia y como una útil guía pronostica de esta patología.

\section{Agradecimientos}

EU. Daniela Calderón, quién colaboró en la toma de muestras para nuestro estudio.

\section{Referencias}

1.- Joint Committee on Infant Hearing: Year 2000 Position Statement: Principles and guidelines for early hearing detection and intervention programs. http://www.cdc. gov/ncbddd/ehdi/documents/jcihyr2000.pdf

2.- American Academy of Pediatrics Task Force on New- born and Infant Screening: Policy Statement. Newborn and Infant hearing loss: detection and intervention. Pediatrics 1999; 103:527-530

3.- Morton NE: Genetic epidemiology of hearing impairment. Ann N Y Acad Sci 1991; 630: 16-31.

4.- $\quad$ Nazar MG, Goycoolea VM, Godoy SJM, et al: Evaluación auditiva neonatal universal: Revisión de 10000 pacientes estudiados, Rev. Otorrinolaringol. Cir. Cabeza Cuello 2009; 69: 93-102.

5.- Norris VW, Arnos KS, Hanks WD, et al: Does universal newborn hearing screening identify all children with GJB2 (Connexin 26) deafness? Penetrance of GJB2 deafness. Ear Hear 2006; 27 (6): 732-41.

6.- Pittaluga E, Godoy C, Bustamante L, San Martín J, Dalbosco D: Screening Auditivo Neonatal Universal: experiencia 4 años en un centro asistencial público. III Congreso Chileno de Neonatologia 2010.

7.- Smith RJ, Bale JF Jr, White KR: Sensorineural hearing loss in children. Lancet 2005; 365: 879-90.

8.- Del Castillo FJ, Rodríguez-Ballesteros M, Álvarez A, et al: Novel deletion involving the connexin-30 gene, del(GJB6-d13s1854), found in trans with mutations in the GJB2 gene (connexin-26) in subjects with DFNB1 non-syndromic hearing impairment. J Med Genet 2005; 42: 588-94.

9.- Gasparini P, Rabionet R, Barbujani G, et al: High carrier frequency of the del35G deafness mutation in European population. Genetic Analysis Consortium of GJB2 35delG. Eur J Hum Genet 2001; 8: 19-23.

10.- Hone SW, Smith RJH: Genetic Screening for Hearing Loss. Clin Otolaryngol Allied Sci 2003; 28 (4): 285-90.

11.- Scott DA, Kraft ML, Carmi R, et al: Identification of Mutations in the Connexin 26 Gene that Cause Autosomal Recessive Nonsyndromic Hearing Loss. Hum Mut 1998; 11: 387-94.

12.- Kudo T, Ikeda K, Kure S, et al: Novel Mutations in the Connexin 26 Gene (GJB2) Responsible for Childhood Deafness in the Japanese Population. Am J Med Genet 2000; 90 (2): 141-5.

13.- Denoyelle F, Marlin S, Weil D, et al: Clinical Features of the Prevalent form of Childhood Deafness, DFNB1, Due to a Connexin-26 Gene Defect: Implications for Genetic Counselling. Lancet 1999; 353 (9161): 1298304.

14.- Denoyelle F, Weil D, Maw MA, et al: Prelingual deafness: high prevalence of a 30delG mutation in the connexin 26 gene, Human Molecular Genetics 1997; 6, (12): 21737.

15.- Dalamón V, Lotersztein $V$, Béhèran $A$, et al: GJB2 and GJB6 genes: molecular study and identification of novel 
GJB2 mutations in the hearing-impaired Argentinean population. Audiol Neurootol 2010; 15 (3): 194-202. Epub 2009 Nov 4.

16.- Gravina LP, Foncuberta ME, Prieto ME, Garrido J, Barreiro $C$, Chertkoff L: Prevalence of DFNB1 mutations in Argentinean children with non-syndromic deafness. Report of a novel mutation in GJB2. Int J Pediatr Otorhinolaryngol 2010; 74 (3): 250-4. Epub 2009 Dec 22.

17.- Utrera $R$, Ridaura $V$, Rodríguez $Y$, et al: Detection of the 35delG/GJB2 and del(GJB6-D13S1830) mutations in Venezuelan patients with autosomal recessive nonsyndromic hearing loss. Genet Test 2007; 11 (4): 347-52.

18.- Snoeckx RL, Huygen PL, Feldmann D, et al: GJB2 mu- tations and degree of hearing loss: a multicenter study Am J Hum Genet 2005; 77 (6): 945-57. Epub 2005 Oct 19.

19.- Ballana E, Ventayol M, Rabionet R, Gasparini P, Estivill $X$ : Connexins and deafness Homepage. World wide web URL: http://www.crg.es/deafness

20.- Thompson DC, McPhillips H, Davis RL, Lieu TL, Homer CJ, Helfand $M$ : Universal newborn hearing screening: summary of evidence. JAMA 2001; 286 (16): 2000-10.

21.- Instituto Mexicano del Seguro Social: Guía de Hipoacusia Neurosensorial Bilateral e Implante Coclear, México; 2010. 